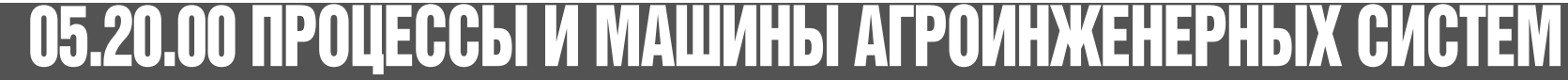

\section{ТЕОРЕТИЧЕСКОЕ ОБОСНОВАНИЕ ЗЕРНОСУШИЛКИ СО СПИРАЛЬНЫМ РАБОЧИМ ОРГАНОМ}

Долгов Владимир Иванович, аспирант кафедры «Агротехнологии, машины и безопасность жизнедеятельности»

Курдюмов Владимир Иванович, доктор технических наук, профессор, заведующий кафедрой "Агротехнологии, машины и безопасность жизнедеятельности»

Павлушин Андрей Александрович, доктор технических наук, профессор кафедры «Агротехнологии, машины и безопасность жизнедеятельности»

ФГБОУ ВО УЛЬЯнОвСКИЙ ГАУ

432017, г. Ульяновск, бульвар Новый Венеи, дом 1; тел.: 89050359200; e-mail: andrejpavlu@ yandex.ru

Ключевые слова: зерно, движение, рабочий орган, параметры, конструкция, спираль Архимеда

Сушка зерна - сложный и ответственный процесс, от результатов которого зависит сохранность полученного урожая. По результатам проведённого анализа существующих конструкций зерносущилок нами выявлено, что они в основном предназначены для сушки продовольственного зерна. Для сушки зерна на селекционные цели в существующих зерносушилках необходимо осуществлять значительные трансформации в их режимных и конструктивных параметрах. Всё это приводит к необоснованным увеличениям сроков обработки зерна и себестоимости процесса теплового воздействия. Нами предложена новая запатентованная конструкция селекционной зерносушилки. Отличием предлагаемого средства механизации является выполнение транспортирующего рабочего органа в виде плоской пружины, на которой установлена жёстко связанная с ней и снабженная приводом перфорированная пластина круглой формы. Нагревательный элемент располагают под транспортирующим рабочим органом и выполняют в виде перфорированного плоского кольца, в отверстиях которого устанавливают втулки, выполненные из теплоизолирующего материала. Вентилятор соединяют воздуховодом с перфорацией нагревательного элемента. Целью проводимых исследований стало теоретическое обоснование параметров разрабатываемого средства механизации. В результате проведённых исследований выявлено влияние градиента температуры в зависимости от толщины слоя высушиваемого зерна. В частности, обосновано, что при контактном способе подвода теплоты нагрев зернового слоя толщиной, превосходящей толщину единичного слоя, протекает медленно, хотя значение коэффициента теплопередачи в данном случае превосходит значение аналогичного показателя при конвективной сушке. Это и предопределяет эфрфективность применения контактного способа передачи теплоты при сушке зерна в единичном слое. Важным фактором при этом является необходимость осуществления постоянного движения и перемешивания зернового слоя, чтобы избежать локальных перегревов зерна, находящегося в непосредственном контакте с греющей поверхностью.

\section{Работа выполняется в рамках гранта Президента Российской Федерации для государственной поддержки молодых российских ученых - докторов наук МД-1673.2018.8}

\section{Введение}

Тепловую обработку продукции растениеводческой отрасли сельского хозяйства широко используют с целью доведения сырья до кондиционного состояния (рис. 1) [1].

Следует отметить, что одним из важных 


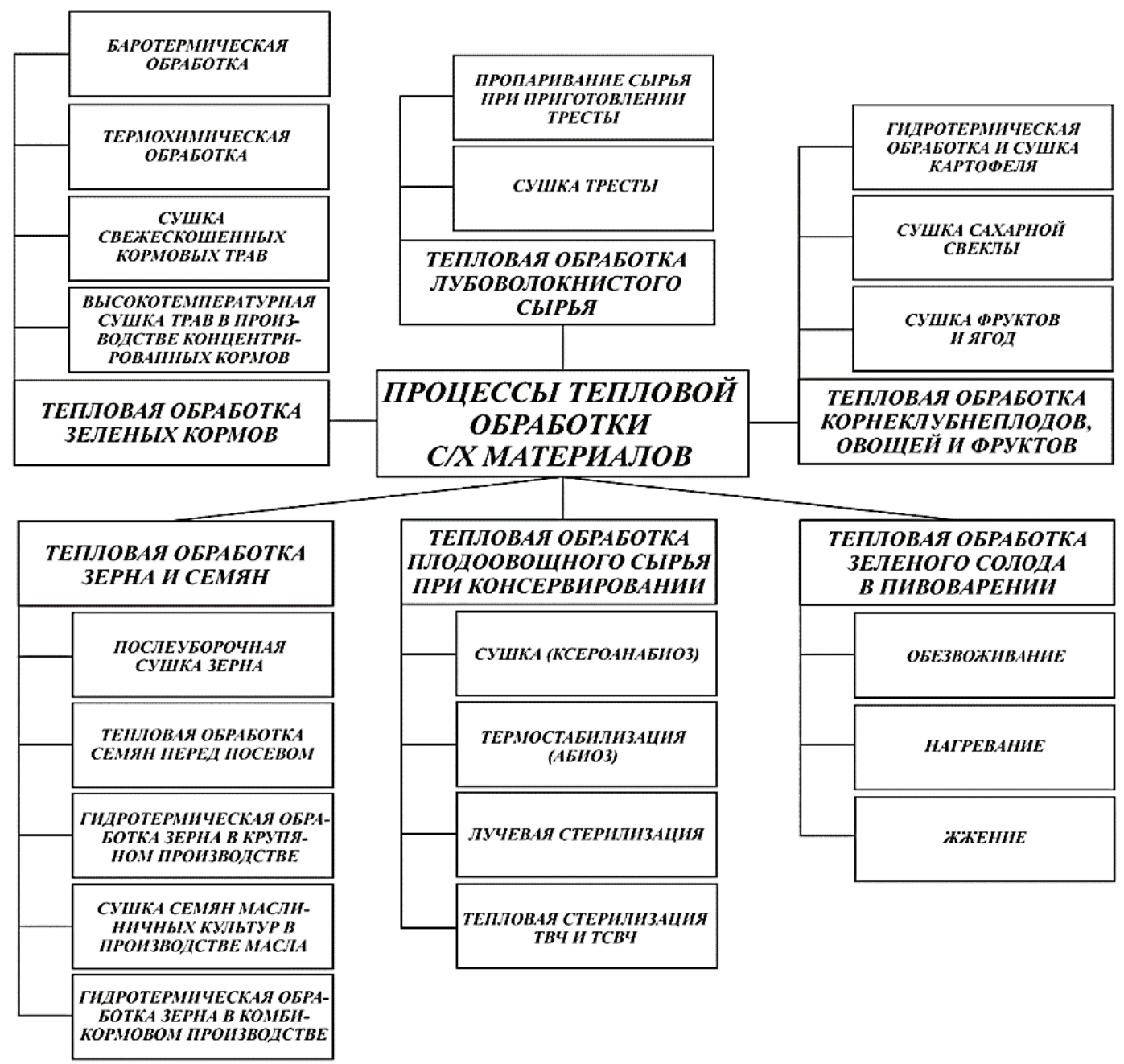

Рис. 1 - Классификация процессов тепловой обработки сельскохозяйственных материалов

назначений теплового воздействия на исходные продукты является снижение влажности зерна до параметров, при которых возможно его длительное хранение без снижения основных качественных критериев [2].

Обеспечить качественную сохранность зерна в процессе его обработки - сложное и дорогостоящее дело, требующее высокоэффективной технологической базы. Разрабатываемые и создаваемые зерносушильные комплексы обеспечивают качественную сушку продовольственного зерна различной влажности [3, 4, 5]. Однако для того, чтобы использовать существующие комплексы по подработке зерна для сушки семян, необходимо их существенно конструктивно доработать. Также требуется внести значительные изменения в режимные параметры технологического процесса. Но даже эти манипуляции не позволяют добиться высококачественной тепловой обработки семян, которая обеспечит их кондиционную всхожесть. В связи с этим актуальным для семеноводческих и экспериментальных зернопроизводящих хозяйств является научное обоснование и создание принципиально новой конструкции зерносушилки, обеспечивающей качественную тепловую сушку зерна семенного назначения.

\section{Объекты и методы исследований}

Для решения поставленной задачи нами предложена новая конструктивная схема селекционной зерносушилки (рисунок 2) [6, 7, 8].

Благодаря встроенным в нагревательный элемент теплоизолированным втулкам, которые соединяют воздуховод зерносушилки с камерой сушки, обеспечивается качественный влагоотвод. При этом воздушный поток не охлаждает нагревательный элемент, что обеспечивает оптимальные энергозатраты на процесс сушки зерна.

Спиральный рабочий орган зерносушил- 


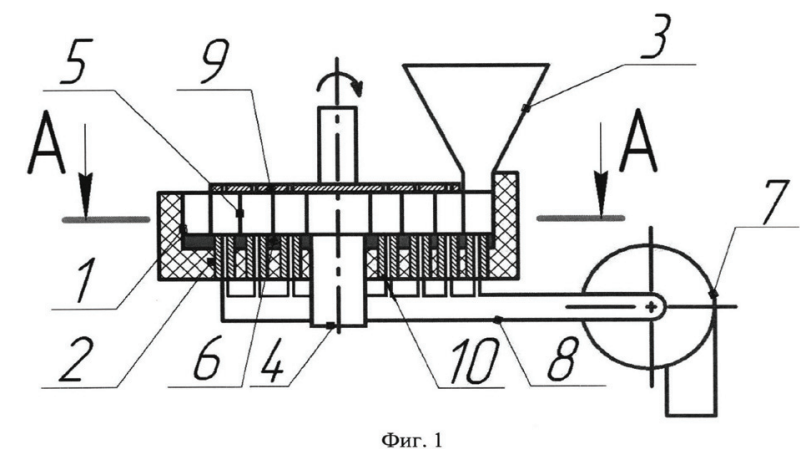

Рис. 2 - Селекционная зерносушилка со спиральным рабочим органом:

1 - кожух; 2 - теплоизолирующий материал; 3 - загрузочный бункер; 4 - выгрузное окно; 5 - спиральный рабочий орган; 6 - нагревательный элемент; 7-вентилятор; 8-воздуховод; 9 - перфорированная пластина; 10 втулки

ки с конструктивными параметрами, позволяющими транспортировать обрабатываемое зерно единичным слоем, обеспечивает высокую равномерность теплового воздействия на обрабатываемый продукт, за счёт чего повышается качество процесса.

\section{Результаты исследований}

Передача теплоты в разрабатываемом средстве механизации осуществляется контактным способом. Тепловая энергия от электрического нагревательного элемента передаётся непосредственно обрабатываемому зерну, находящемуся в сушильной камере зерносушилки и перемещающемуся от загрузочного бункера к выгрузному окну с помощью рабочего органа, выполненного в виде спирали Архимеда.

Рассматриваемый механизм подвода теплоты можно интерпретировать, с определёнными упрощениями, как передачу теплоты посредством теплопроводности $[9,10]$.

При этом изменение потока теплоты при сушке зерна в разрабатываемом средстве механизации зависит от накопленной тепловой энергии в рассматриваемом объёме высушиваемого зерна, транспортируемом в межвитковом пространстве спирального рабочего органа:

$\left[\frac{\partial}{\partial x}\left(\lambda \frac{\partial t}{\partial x}\right)+\frac{\partial}{\partial y}\left(\lambda \frac{\partial t}{\partial y}\right)+\frac{\partial}{\partial z}\left(\lambda \frac{\partial t}{\partial z}\right)\right] d x, d y, d z$,

где $\lambda$ - коэффициент теплопроводности, $\mathrm{BT} /\left(\mathrm{M} \cdot{ }^{\circ} \mathrm{C}\right)$.

Энергетический баланс для объёма высушиваемого зерна, находящегося в сушильной камере, может быть сформулирован в следующем виде:

$c \rho \frac{\partial t_{\text {Hэ }}}{\partial \tau}=\frac{\partial}{\partial x}\left(\lambda \frac{\partial t_{H \ni}}{\partial x}\right)+\frac{\partial}{\partial y}\left(\lambda \frac{\partial t_{\text {нэ }}}{\partial y}\right)+\frac{\partial}{\partial z}\left(\lambda \frac{\partial t_{H \ni}}{\partial z}\right)+q$,

где $C$ - теплоёмкость нагревательного элемента, кДж/(кг $\left.{ }^{\circ} \mathrm{C}\right) ; \rho$ - теплота парообразова-

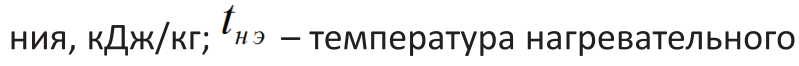
элемента, ${ }^{\circ} \mathrm{C} ; \tau$ - время нахождения высушиваемого зерна в сушильной камере устройства, с; $q=c \rho d x d y d z \frac{\partial t}{\partial \tau}$ $\partial \tau$ - количество теплоты, передаваемой от поверхности нагревательного элемента зерносушилки к высушиваемому объёму зерна, кДж.

При этом систему дифференциальных уравнений передачи теплоты от нагревательного элемента зерносушилки к контактирующему с ней зерну можно смоделировать в виде [11]:

$$
\left.\begin{array}{c}
\frac{\partial t_{н э}(\xi, x)}{\partial \tau}=a\left[\frac{\partial^{2} t_{\mu э}(\xi, x)}{\partial \xi^{2}}\right]+\frac{\varepsilon \rho \partial \Theta(\xi, x)}{\partial \tau}, \\
\frac{\partial \Theta(\xi, x)}{\partial \tau}=a\left[\frac{\partial^{2} \Theta(\xi, x)}{\partial \xi^{2}}\right], \\
\frac{\partial t_{3}(0, \tau)}{\partial \xi}=0, t_{н э}\left(t_{1}, t_{2}\right) \leq \infty \\
\frac{\partial \Theta(0, \tau)}{\partial \xi}=0, \Theta\left(t_{3}, \tau\right) \leq \infty
\end{array}\right\},
$$

где а - коэффициент температуропроводности, $\mathrm{M}^{2} / \mathrm{c} ; \varepsilon$ - критерий фазового перехода; $\xi$ - коэффициент, характеризующий область распространения зоны испарения, М; $\Theta=\left[t_{(0, \tau)}-t_{H \ni}\right]$ - разность между варьирующей температурой высушиваемого зерна $t_{3}$ и постоянной температурой нагревательного элемента $t_{\text {нэ }, ~}{ }^{\circ} \mathrm{C} ; \mathrm{t}_{1}, \mathrm{t}_{2}$ - начальная и рабочая температура нагревательного элемента зерносушилки соответственно, ${ }^{\circ} \mathrm{C}$.

При движении зерна спиральным рабочим органом в предлагаемом средстве механизации создаются равные тепловые характеристики для всех обрабатываемых зёрен [12, 13, 14]: 
$\frac{t_{H \ni}-t_{32}}{t_{H \rho}-t_{31}}=\exp \left(-\frac{a_{m} f_{G}}{c \rho} \tau\right)+\frac{k_{c} \frac{r}{c}\left(\omega_{H}-\omega_{\kappa}\right)}{\left(\frac{a_{m} f_{G}}{c \rho}-k_{c}\right)\left(t_{\text {нэ }}-t_{31}\right)} \exp \left[\left(-\frac{a_{m} f_{G}}{c \rho}-k_{c}\right) \tau\right]$,

где $t_{32}$ и $t_{31}$ - температура зерна до $и$ после сушки в селекционной зерносушилке соответственно, ${ }^{\circ} \mathrm{C} ; a_{m}$ - коэффициент теплооб-

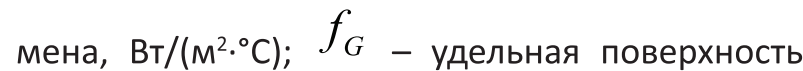
зерна, $\mathrm{M}^{2} /$ кг; с - удельная теплоёмкость высушиваемого зерна, Дж/(кг $\left.{ }^{\circ} \mathrm{C}\right) ; \rho$ - удельная теплота фазового перехода, Дж/кг; $k_{c}$ - коэффициент сушки; r- теплота парообразования, Дж/ кг; $\omega_{H}, \omega_{\kappa}$ - начальная и конечная влажности зерна соответственно, \%.

Решение системы уравнений 3 имеет вид: $\frac{\partial \Theta_{(z, \tau)}}{\partial z}=\frac{a_{m}}{\lambda}\left(t_{\mu э}-t_{3}\right) \exp \left[\frac{a_{m}}{\lambda}\left(z+\frac{a_{m}}{\lambda}\right)\right]\left[1-\operatorname{erf}\left(\frac{z}{2 \sqrt{a \tau}}+\frac{a_{m}}{\lambda} \sqrt{a \tau}\right)\right]$,

где z - толщина высушиваемого слоя зерна в межвитковом пространстве спирального рабочего органа зерносушилки, м.

Изменение градиента температуры нагрева обрабатываемого зерна во времени и по толщине обрабатываемого слоя, интерпретируемого зависимостью (5), представлено на рисунке 3.

Градиент температуры высушиваемого зерна достигает своего предельного значения в первый период у зернового слоя, непосредственно контактирующего с нагревательным элементом, и затем снижается в последующих слоях зерна [15].

Следовательно, сушка зерна в его непосредственном контакте с нагревательным элементом при движении зернового слоя толщиной, значительно превышающей максимальные размеры единичной зерновки, протекает длительно, хотя коэффициент теплопередачи в этом случае существенно превосходит значение аналогичного показателя при конвекционном подводе теплоты. Это и подтверждает преимущество применения контактного способа передачи теплоты при сушке зерна слоем, толщина которого незначительно превосходит толщину единичного слоя. Важно при перемещении зерна спиральным рабочим органом обеспечить его качественное перемешивание. В этом случае минимизируется местный перегрев обрабатываемого зерна, расположенного в непосредственном контакте с поверхностью нагревательного элемента, что и обеспечивается в предлагаемом средстве механизации.

\section{Выводы}

При послеуборочной обработке для обеспечения основных качественных показателей

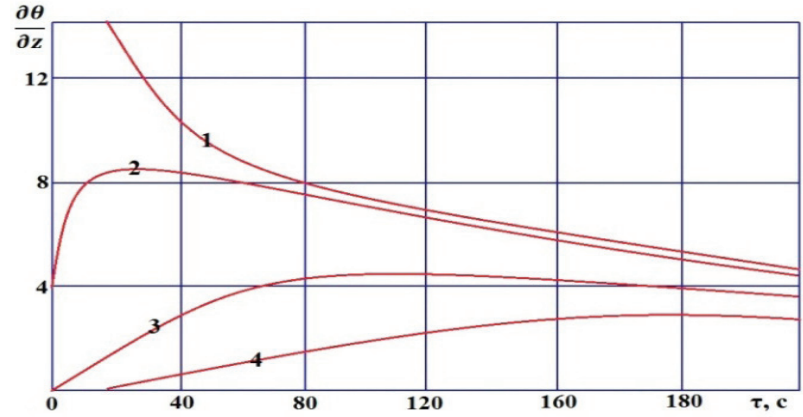

Рис. 3 - Изменение градиента температуры нагрева зерна при подводе теплоты от контактирующей поверхности:

$4-(z=75 \mathrm{MM})$

1 - $(z=0$ мM); 2 - (z = $15 \mathrm{Mm}) ; 3$ - (z = $45 \mathrm{MM}) ;$

зерна важно грамотно осуществить процесс сушки. При этом необходимо обеспечить высокую равномерность обработки высушиваемого зерна при отсутствии локального перегрева отдельных зёрен. Решение указанной задачи возможно при использовании зерносушилки со спиральным транспортирующим рабочим органом. Техническим результатом при применении предлагаемого средства механизации является снижение удельных затрат энергии в 1,3...1,6 раза по сравнению с серийно выпускаемыми зерносушилками конвективного типа при обеспечении требуемого качества высушенного зерна.

\section{Библиографический список}

1. Трисвятский, Л.А. Технология приема, обработки, хранения зерна и продуктов его переработки / Л.А.Трисвятский, Б.Е.Мельник. - М.: Колос, 1983. - $351 \mathrm{c}$.

2. Государственная программа развития сельского хозяйства и регулирования рынков сельскохозяйственной продукции, сырья и продовольствия на 2013-2020 годы. - М.: МСХ РФ, 2012. - 204 с.

3. Vargas, W.L. Heat conduction in granular materials / W.L.Vargas, J.J. McCarthy // AIChE Journal. - 2001. - № 47. - P. 1052 - 1059.

4. Wang, L.J. Rapid cooling of porous and moisture foods by using vacuum cooling technology / L.J.Wang, D.W. Sun // Trends in Food Science Technology. - 2001. - № 12. - P. 174 - 184.

5. Yadollahinia, A.R. Design and fabrication of experimental dryer for studying agricultural products / A.R.Yadollahinia, M.Omid, S.Rafie // Int. J. Agri. Biol. - 2008. - № 10. - P. 61 - 65.

6. Пат. 171703 Российская Федерация, МПК F26B 15/04. Устройство для сушки зерна / В.И. Курдюмов, А.А. Павлушин, Г.В. Карпенко, С.А. Сутягин, В.И. Долгов, П.С. Агеев; патентообладатель ФгОУ ВО Ульяновская ГСХА. - № 2016131812; заявл. 02.08.2016; опубл. 13.06.2017, Бюл. № 17.

7. Пат. 96639 Российская Федерация, МПК F26B 3/00. Устройство для сушки зерна / В.И. 
Курдюмов, А.А. Павлушин, И.А. Постников; патентообладатель ФГОУ ВО Ульяновская ГСХА. - № 2010106454/22; заявл. 24.02.2010; опубл. 10.08.2010, Бюл. № 22.

8. Пат. 2453123 Российская Федерация, МПК А23В 9/08. Устройство для сушки зерна / В.И. Курдюмов, А.А. Павлушин, С.А. Сутягин; патентообладатель ФГОУ ВО Ульяновская ГСХА.№ 2010145902/13; заявл. 10.11.2010; опубл. 20.06.2012, Бюл. № 17.

9. Беляев, Н.М. Основы теплопередачи / Н.М. Беляев. - Киев: Высш. школа, 1989. - 342 с.

10. Курдюмов, В.И. Теоретическое обоснование динамики сушки зерна при контактном способе теплоподвода / В.И.Курдюмов, А.А.Павлушин, Г.В. Карпенко // Вестник Ульяновской государственной сельскохозяйственной академии. - Ульяновск, 2015. - № 3 (31). - С. 125 - 130.
11. Влияние параметров зерносушильной установки на качество сушки / В.И. Курдюмов, А.А. Павлушин, Г.В. Карпенко, С.А Сутягин // Доклады Российской академии сельскохозяйственных наук. - 2012. - № 6. - С. 74 - 76.

12. Лыков, А.В. Теория тепло - и массопереноса / А.В.Лыков, Ю.А.Михайлов. - М.-Л.: Госэнергоиздат, 1963. - 536 с.

13. Лыков, А.В. Теория теплопроводности / А.В. Лыков. - М.: Высш. школа, 1967. - 599 с.

14. Баум, А.Е. Сушка зерна / А.Е. Баум, B.A. Резчиков. - М.: Колос, 1983. - 223 с.

15. Савченко, Светлана Вениаминовна. Развитие научных основ и практических методов повышения эффективности технологии зерносушения: дис. ... д-ра технических наук: 05.18.01 / С.В. Савченко. - Москва, 2009. - 387 с.

\title{
THEORETICAL SUBSTANTIATION OF THE GRAIN DRYER WITH A SPIRAL WORKING ELEMENT
}

\author{
Dolgov V.I., Kurdyumov V.I., Pavlushin A.A. \\ FSBEI HE Ulyanovsk SAU \\ 432017, Ulyanovsk, Noviy Venets Boulevard, 1; \\ tel.: 89050359200; e-mail: andreipavlu@yandex.ru.
}

Key words: grain, movement, working element, parameters, construction, Archimedes' spiral.

Grain drying is a complex and essential process, the safety of crop depends on the results of that process. Based on the results of the analysis of existing designs of grain dryers, we found that they mainly aim at drying food grain. For grain drying for selection purposes, it is necessary to carry out significant transformations in their regime and design parameters of existing grain dryers. It leads to unreasonable increase in time of grain treatment and cost of heat treatment process. We have proposed a new patented design of a selection grain dryer. The difference of the proposed means of mechanization is the transporting working element in the form of a flat spring which has a perforated plate of a circular shape and equipped with a drive rigidly connected to it. The heating element is placed under the transporting working element and is made in the form of a perforated flat ring, the holes of which have bushings made of heat-insulating material. The fan is connected by an air duct to the perforation of the heating element. The aim of the research was the theoretical substantiation of the parameters of the devised means of mechanization. As a result of the research, the influence of the temperature gradient which depends on the thickness of the dried grain was detected. In particular, it was justified that, in the contact method of heat supplying, the heating of a grain layer thicker than the thickness of a single layer goes slowly, although the value of the heat transfer coefficient in this case exceeds the value of a similar parameter for convective drying. This predetermines the effectiveness of application of the contact method of heat transfer when drying grain in a single layer. An important factor is the need for constant movement and mixing of the grain layer to avoid local overheating of the grain, which is in direct contact with the heating surface.

Bibliography

1. Trisvyatsky, L.A. Technology of receiving, processing, storage of grain and products of its processing / L.A. Trisvyatsky, B.E. Melnik. - Moscow: Kolos, 1983. $-351 p$.

2. State program of development of agriculture and regulation of markets of agricultural products, raw materials and food for 2013-2020. - Moscow: Ministry of Agriculture of the Russian Federation, 2012. - 204 p.

3. Vargas, W.L. Heat conduction in granular materials / W.L.Vargas, J.J. McCarthy // AlChE Journal. - 2001. - No $47 .-$ P. 1052 - 1059.

4. Wang, L.J. Rapid cooling of porous and moisture foods by using vacuum cooling technology / L.J.Wang, D.W. Sun //Trends in Food Science Technology. -2001. - № 12. - P. 174 - 184.

5. Yadollahinia, A.R. Design and fabrication of experimental dryer for studying agricultural products / A.R.Yadollahinia, M.Omid, S.Rafie // Int. J. Agri. Biol. - 2008. - № 10. - P. 61 - 65.

6. Pat. 171703 Russian Federation, IPC F26B 15/04. Device for grain drying / V.I. Kurdyumov, A.A. Pavlushin, G.V. Karpenko, S.A. Sutyagin, V.I. Dolgov, P.S. Ageev; patent owner FSBEI HE Ulyanovsk State Agricultural Academy. - appl. 02.08.2016; publ. 13.06.2017, Bul. №. 17.

7. Pat. 96639 Russian Federation, IPC F26B 3/00. Device for grain drying / V.I. Kurdyumov, A.A. Pavlushin, I.A. Postnikov; patent FSBEI HE Ulyanovsk State Agricultural Academy. - appl. 24.02.2010; publ. 10.08.2010, Bul. № 22.

8. Pat. 2453123 Russian Federation, IPC A23B 9/08. Device for grain drying / V.I. Kurdyumov, A.A. Pavlushin, S.A. SUtyagin; patent owner FSBEI HE Ulyanovsk State Agricultural Academy.- appl 10.11.2010; publ. 20.06.2012, Bul. № 17.

9. Belyaev, N.M. Fundamentals of heat transfer / N.M. Belyaev. - Kiev: Vyshaya Shkola, 1989. - 342 p.

10. Kurdyumov, V.I. Theoretical substantiation of grain drying dynamics in case of contact method of heat supply / V.I. Kurdyumov, A.A. Pavlushin, G.V. Karpenko // Vestnik of Ulyanovsk State Agricultural Academy. - Ulyanovsk, 2015. - № 3 (31). - P. 125 - 130.

11. Influence of grain drying device parameters on quality of drying / V.I. Kurdyumov, A.A. Pavlushin, G.V. Karpenko, S.A. Sutyagin // Reports of the Russian Academy of Agricultural Sciences. - 2012. - № 6. - P. 74 - 76.

12. Lykov, A.V. Theory of heat and mass transfer / A.V. Lykov, Yu.A. Mikhailov. - M.-L .: Gosenergoizdat, 1963. - 536 p.

13. Lykov, A.V. Theory of heat conductivity / A.V. Lykov. - M.: Vyshaya Shkola, 1967. - 599 p.

14. Baum, A.E. Grain drying / A.E. Baum, V.A. Rezchikov - Moscow: Kolos, 1983. - 223 p.

15. Savchenko, Svetlana Veniaminovna. Development of scientific foundations and practical methods for improving the efficiency of grain drying technology: dissertation of Doctor of Technical sciences: 05.18.01 /S.V. Savchenko. - Moscow, 2009. - 387 p. 